\title{
Single Crystalline Oxygen-free Titanium Nitride by XPS
}

Dominik Jaeger ${ }^{\text {a) }}$ and Jörg Patscheider ${ }^{\text {a) }}$

Empa, Swiss Federal Laboratories for Materials Science and Technology, Laboratory for

Nanoscale Materials Science, Überlandstrasse 129, 8600 Dübendorf, Switzerland

(Received 2 November 2012; accepted 5 February 2013; published 5 March 2013)

X-ray photoelectron spectroscopy (XPS) spectra are presented, which are obtained from an oxygen-free single crystalline (sc-) titanium nitride (TiN) sample. The investigated film has been grown on a magnesium oxide ( $\mathrm{MgO})$ single crystal with the (001) orientation. Unbalanced Reactive Magnetron Sputter deposition was used to deposit the TiN film in an argon/nitrogen atmosphere at $5 \times 10^{-3} \mathrm{mbar}$ and a temperature of $800^{\circ} \mathrm{C}$. The sample has been transferred in situ from the deposition chamber to the XPS device in order to prevent surface oxidation of the sample. Atomic force microscopy (AFM), X-ray diffraction (XRD), Rutherford backscattering (RBS) and angle resolved (AR-) XPS have been used to characterize the sample in detail. This work is dedicated to the XPS characterization of a representative oxygen-free sc-TiN sample. Detailed scans are presented and discussed for the Ti 2p, O 1s, N 1s, Ti 2s, valence band and Ti LMM regions. The spectra contain shake-ups, surface and bulk plasmons, that can be separated and quantified by the presented evaluation procedure. (C) 2013 American Vacuum Society.

[http://dx.doi.org/10.1116/11.20121107]

Keywords: titanium nitride; shake-up; satellite; Tougaard background

PACS: 81.15.Cd, 82.80.Yc, 61.05.cp, 73.20.At, 73.20.Mf, 79.60.Dp

\section{INTRODUCTION}

Titanium nitride (TiN) is often used as a protective and decorative coating. There is extensive literature available on XPS spectra of pure TiN (Refs. 1-5 and further references therein). The Ti $2 p$ spectrum of TiN contains, besides the well-known two spin-orbit split peaks Ti $2 \mathrm{p}_{1 / 2}$ and Ti $2 \mathrm{p}_{3 / 2}$, also shake-ups as well as surface and bulk plasmons. The quantification and the interpretation of the shake-ups have been subject of discussion in the aforementioned papers and have only recently been quantified (Ref. 1). In the present contribution XPS data of a pristine oxygen-free sc-TiN sample are shown and a selfconsistent data evaluation recommendation is presented.

\section{SPECIMEN DESCRIPTION (ACCESSION \#01247)}

Host Material: TiN (001)

CAS Registry \#: 25583-20-4

Host Material Characteristics: homogeneous; solid; single crystal; conductor; inorganic compound; coating

Chemical Name: titanium nitride

Source: See History and Significance

Host Composition: TiN

Form: TiN (001) deposited at Empa, Dübendorf

Lot \#: 1768 (internal experiment \#)

Structure: [ $\left.\begin{array}{lll}0 & 0 & 1\end{array}\right]$

History / Significance: Titanium nitride is fabricated by unbalanced magnetron sputtering, where a pure titanium target (99.99\%) is used to deposit TiN in an argon-nitrogen atmosphere. The Ar gas has a quality of 6.0 and $\mathrm{N}_{2}$ of 5.0. The crystallinity of TiN has been

a) Author to whom correspondence should be addressed. checked by recording pole figures using XRD. RBS measurements show an oxygen concentration in the bulk of less than 0.2 at $\%$. AFM results indicate a roughness $S_{\mathrm{q}}$ of approximately $0.5 \mathrm{~nm}$.

As Received Condition: The sample has been studied as deposited and has been transferred in situ ( $\left.\mathrm{p}<5 \times 10^{-10} \mathrm{mbar}\right)$ from the deposition chamber to the XPS device.

Analyzed Region: not specified

Ex Situ Preparation/Mounting: A Pt-frame has been deposited on a $\mathrm{MgO}$ crystal (001). The Pt-frame functions as an electrical contact. Additionally Mo-bands were used to mount the MgOsample on a Mo-sample holder.

In Situ Preparation: A TiN film has been deposited on top of this setting ( $\mathrm{MgO}$ crystal with Pt-frame) before transferring the sample in situ to the XPS device. No sputter cleaning or annealing has been performed.

Pre-Analysis Beam Exposure: The sample has been exposed to $\mathrm{X}$-rays up to $24 \mathrm{~h}$.

Charge Control: none

Temp. During Analysis: $300 \mathrm{~K}$

Pressure During Analysis: $<5 \times 10^{-7} \mathrm{~Pa}$

\section{INSTRUMENT DESCRIPTION}

Manufacturer and Model: Phi-Instrument Quantum 2000

Analyzer Type: spherical sector

Detector: multichannel detector

Number of Detector Elements: 16

INSTRUMENT PARAMETERS COMMON TO ALL SPECTRA -

\section{Spectrometer}

Analyzer Mode: constant pass energy 
Throughput $\left(T=E^{N}\right): \mathrm{N}=0$

Excitation Source Window: none

Excitation Source: $\mathrm{Al} K_{\alpha}$, monochromatic

Source Energy: $1486.6 \mathrm{eV}$

Source Strength: $25 \mathrm{~W}$

Source Beam Size: $100 \mu \mathrm{m} \times 100 \mu \mathrm{m}$

Signal Mode: multichannel direct

Geometry

Incident Angle: 0 or $1^{\circ}$

Source to Analyzer Angle: $45^{\circ}$

Emission Angle: 45 or $44^{\circ}$

Specimen Azimuthal Angle: $0^{\circ}$

Acceptance Angle from Analyzer Axis: not specified

\section{DATA ANALYSIS METHOD}

Energy Scale Correction: To eliminate surface effects (e.g. due to contamination) all binding energies are obtained by using an exponentially decaying fit function that extrapolates angle resolved XPS data to obtain the bulk value for sc-TiN. For a detailed description of the fitting routine it, see Ref. 1. The presented values are binding energies that were measured and extrapolated for four different TiN samples fabricated using the same deposition parameters. Those four samples were prepared and measured independently from each other within a time period of seven months. The presented error is the standard deviation of the interpolated binding energies of these four sc-TiN samples.

Peak Shape and Background Method: All peak shapes are fitted using a least square fitting procedure with a function described by a Gaussian-Lorentzian product (GL(30), for details see Refs. 1 and 6). The background used is a universal-two para- meter Tougaard (U2T) background, where $\mathrm{C}$ has been chosen to be fixed to $\mathrm{C}=355.0 \mathrm{eV}^{2}$ and $\mathrm{B}$ has been allowed to adjust to the limits on the high binding energy side of the selected peak region (for details see Refs. 1 and 6).

Quantitation Method: After the background removal the peak areas have been corrected using the sensitivity factors according to the first principle method (considering Scofield's cross section [Ref. 7], asymmetry function [Ref. 8], transmission function and the inelastic mean free path).

\section{ACKNOWLEDGMENTS}

The financial support by the Swiss National Science Foundation under grants 200021-116392 and 200020-130416 / 1 is gratefully acknowledged.

\section{REFERENCES}

1. D. A. Jaeger and J. Patscheider, J. Electron Spectrosc. Relat. Phenom. 185, 523-534 (2012).

2. R. T. Haasch, T.-Y. Lee, D. Gall, J. E. Greene, and I. Petrov, Surf. Sci. Spectra 7, 197 (2000).

3. I. Bertóti, M. Mohai, J. L. Sullivan, and S. O. Saied, Appl. Surf. Sci. 84, 357 (1995).

4. I. Milošev, H.-H. Strehblow, B. Navinšek, and M. MetikošHuković, Surf. Interface Anal. 23, 529 (1995).

5. L. Porte, L. Roux, and J. Hanus, Phys. Rev. B 28, 3214 (1983).

6. N. Fairley, The Casa Cookbook, Vol. Part 1 (Acolyte Science, High Legh,UK, 2005), Chap. Line Shapes and Background, pp. 147-167.

7. J. H. Scofield, J. Electron Spectrosc. Relat. Phenom. 8, 389 (1976).

8. R. F. Reilman, A. Msezane, and S. T. Manson, J. Electron Spectrosc. Relat. Phenom. 8, 129 (1976). 


\begin{tabular}{|c|c|c|c|c|c|c|c|}
\hline \multicolumn{8}{|c|}{ SPECTRAL FEATURES TABLE } \\
\hline $\begin{array}{l}\text { Spectrum } \\
\text { ID\# }\end{array}$ & $\begin{array}{l}\text { Element/ } \\
\text { Transition }\end{array}$ & $\begin{array}{c}\text { Peak } \\
\text { Energy } \\
(\mathrm{eV})\end{array}$ & $\begin{array}{c}\text { Peak } \\
\text { Width } \\
\text { FWHM (eV) }\end{array}$ & $\begin{array}{l}\text { Peak Area } \\
(\text { eV.cts/s) }\end{array}$ & $\begin{array}{l}\text { Sensitivity } \\
\text { Factor }\end{array}$ & $\begin{array}{c}\text { Concentration } \\
\text { (at. \%) }\end{array}$ & Peak Assignment \\
\hline 01247-03 & $\mathrm{Ti} 2 \mathrm{p}_{3 / 2}$ & 455.0 & 1.3 & $\sim 5000$ & 5.7 & 8.2 & Ti-N \\
\hline $01247-03^{a}$ & $\mathrm{Ti} 2 \mathrm{p}_{3 / 2}$ & 457.2 & 3.8 & $\sim 11000$ & 5.7 & 18.0 & Ti-N, shake-up \\
\hline 01247-03 & $\mathrm{Ti} 2 \mathrm{p}_{1 / 2}$ & 460.9 & 1.3 & $\sim 2600$ & 2.9 & 8.2 & Ti-N \\
\hline $01247-03^{a}$ & $\mathrm{Ti} 2 \mathrm{p}_{1 / 2}$ & 462.6 & 3.8 & $\sim 5700$ & 2.9 & 18.0 & Ti-N, shake-up \\
\hline 01247-03 & $\mathrm{Ti} 2 \mathrm{p}_{3 / 2}$ & 467.5 & 7.3 & $\sim 3500$ & 5.7 & 5.8 & Ti-N, surface plasmon \\
\hline 01247-03 & $\mathrm{Ti} 2 \mathrm{p}_{1 / 2}$ & 474.0 & 7.3 & $\sim 1700$ & 2.9 & 5.4 & Ti-N, surface plasmon \\
\hline 01247-03 & Ti $2 p$ & 481.4 & 10.5 & $\sim 4000$ & 8.5 & 4.4 & Ti-N, first bulk plasmon \\
\hline 01247-03 & $\operatorname{Ti} 2 p$ & 507.2 & 10.6 & $\sim 1300$ & 8.3 & 1.5 & Ti-N, second bulk plasmon \\
\hline 01247-04 & $\mathrm{N} 1 \mathrm{~s}$ & 397.1 & 1.1 & $\sim 5000$ & 1.9 & 25 & $\mathrm{~N}-\mathrm{Ti}$ \\
\hline $01247-04^{b}$ & $\mathrm{~N} 1 \mathrm{~s}$ & 399.5 & 2.3 & $\sim 700$ & 1.9 & 3.4 & $\mathrm{~N}-\mathrm{O}$ \\
\hline $01247-05^{c}$ & $01 \mathrm{~s}$ & 530.8 & 2.2 & $\sim 400$ & 2.8 & 1.3 & $\mathrm{O}-\mathrm{Ti}$ \\
\hline $01247-05^{d}$ & $01 \mathrm{~s}$ & 533.0 & 2.2 & $\sim 300$ & 2.8 & 0.9 & $\mathrm{O}-\mathrm{N}$ \\
\hline
\end{tabular}

${ }^{a}$ To obtain a correct shake-up to main peak ratio the reader is referred to Ref. 1.

${ }^{\mathrm{b}}$ This peak at $399.49 \pm 0.11 \mathrm{eV}$ is assigned to N-O bonds; it cannot be due to a surface or a bulk plasmon. The surface plasmon in TiN has an energy of $12.7 \mathrm{eV}$ (see Ti $2 \mathrm{p}$ spectrum), which is about 5 times higher than the difference between the two peaks in $\mathrm{N} 1 \mathrm{~s}(2.4 \mathrm{eV})$.

${ }^{c}$ Peak energy and FWHM have been obtained by extrapolating the data of more than 200 spectra, for details see Ref. 1.

${ }^{\mathrm{d}}$ See annotation of $\mathrm{O} 1 \mathrm{~s}(\mathrm{O}-\mathrm{Ti})$.

\begin{tabular}{lccccccc}
\hline \multicolumn{7}{c}{ ANALYZER CALIBRATION TABLE } \\
\hline $\begin{array}{l}\text { Spectrum } \\
\text { ID\# }\end{array}$ & $\begin{array}{c}\text { Element/ } \\
\text { Transition }\end{array}$ & $\begin{array}{c}\text { Peak } \\
\text { Energy } \\
(\mathbf{e V})\end{array}$ & $\begin{array}{c}\text { Peak } \\
\text { Width } \\
\text { FWHM (eV) }\end{array}$ & $\begin{array}{c}\text { Peak } \\
\text { Area } \\
(\mathbf{e V} \cdot \mathbf{c t s} / \mathbf{s})\end{array}$ & $\begin{array}{c}\text { Sensitivity } \\
\text { Factor }\end{array}$ & $\begin{array}{c}\text { Concentration } \\
\text { (at. \%) }\end{array}$ & $\begin{array}{c}\text { Peak } \\
\text { Assignment }\end{array}$ \\
\hline $9^{\mathrm{a}}$ & $\mathrm{Au} 4 \mathrm{f}_{7 / 2}$ & 84.0 & 0.8 & $\ldots$ & $\ldots$ & $\ldots$ & $\ldots$ \\
$10^{\mathrm{a}}$ & $\mathrm{Ag} 3 \mathrm{~d}_{5 / 2}$ & 368.2 & 0.7 & $\ldots$ & $\ldots$ & $\ldots$ & $\ldots$ \\
$11^{\mathrm{a}}$ & $\mathrm{Cu} 2 \mathrm{p}_{3 / 2}$ & 932.7 & 1 & $\ldots$ & $\ldots$ & $\ldots$ & $\ldots$ \\
\hline
\end{tabular}

${ }^{a}$ The instrument has been calibrated on a regular time basis by measuring the binding energies of $\mathrm{Au} 4 \mathrm{f}_{7 / 2}, \mathrm{Ag} 3 \mathrm{~d}_{5 / 2}$ and $\mathrm{Cu} 2 \mathrm{p}_{3 / 2}$ as well as the transmission function.

\begin{tabular}{lccccc}
\hline \multicolumn{5}{c}{ GUIDE TO FIGURES } \\
\hline $\begin{array}{l}\text { Spectrum } \\
\text { (Accession) \# }\end{array}$ & $\begin{array}{c}\text { Spectral } \\
\text { Region }\end{array}$ & $\begin{array}{c}\text { Voltage } \\
\text { Shift }\end{array}$ & Multiplier & Baseline & Comment \# \\
\hline $1247-01$ & survey & 0 & 1 & 0 & \\
$1247-02$ & Ti $2 \mathrm{p}$ & 0 & 1 & 0 & raw spectrum \\
$1247-02$ & Ti $2 \mathrm{p}$ & 0 & 1 & 0 & fitted spectra \\
$1247-03$ & $\mathrm{~N} 1 \mathrm{~s}$ & 0 & 1 & 0 & \\
$1247-04$ & O 1s & 0 & 1 & 0 & \\
$1247-05$ & valence band & 0 & 1 & 0 & \\
$1247-06$ & Ti 2s & 0 & 1 & 0 & \\
$1247-07$ & Ti LMM & 0 & 1 & 0 & \\
\hline
\end{tabular}

*Voltage shift of the archived (as-measured) spectrum relative to the printed figure. The figure reflects the recommended energy scale correction due to a calibration correction, sample charging, flood gun, or other phenomenon. 


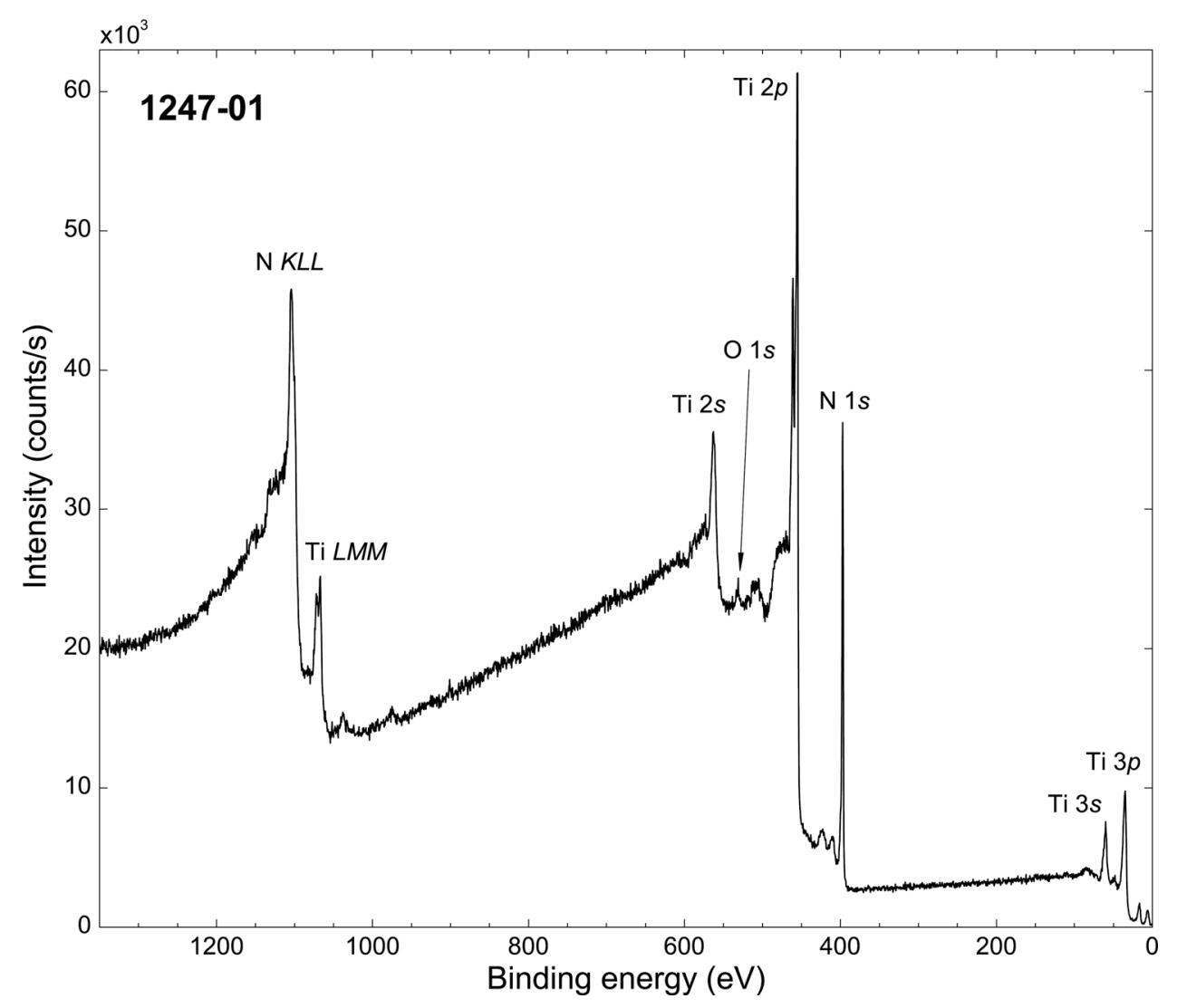

\begin{tabular}{rc}
\hline Accession \# & 01247-01 \\
Host Material & TiN (001) \\
Technique & XPS \\
Spectral Region & survey \\
Instrument & Pxcitation Source \\
Source Energy & Phi-Instrument Quantum 2000 \\
Source Strength & Al $K_{\alpha}$ monochromatic \\
Source Size & $1486.6 \mathrm{eV}$ \\
Analyzer Type & $25 \mathrm{~W}$ \\
Incident Angle & $0.1 \mathrm{~mm} \times 0.1 \mathrm{~mm}$ \\
Emission Angle & spherical sector \\
Analyzer Pass Energy: & 0 or $1^{\circ}$ \\
Analyzer Resolution & $45 \mathrm{or} 44^{\circ}$ \\
Total Elapsed Time & $117.4 \mathrm{eV}$ \\
Number of Scans & $0.5 \mathrm{eV}$ \\
Effective Detector Width & $\sim 1200 \mathrm{~s}$ \\
Total Signal Accumulation Time & $\sim 1260 \mathrm{~s}$ \\
Analyzer Angular Acceptance Width & 6 \\
\hline
\end{tabular}



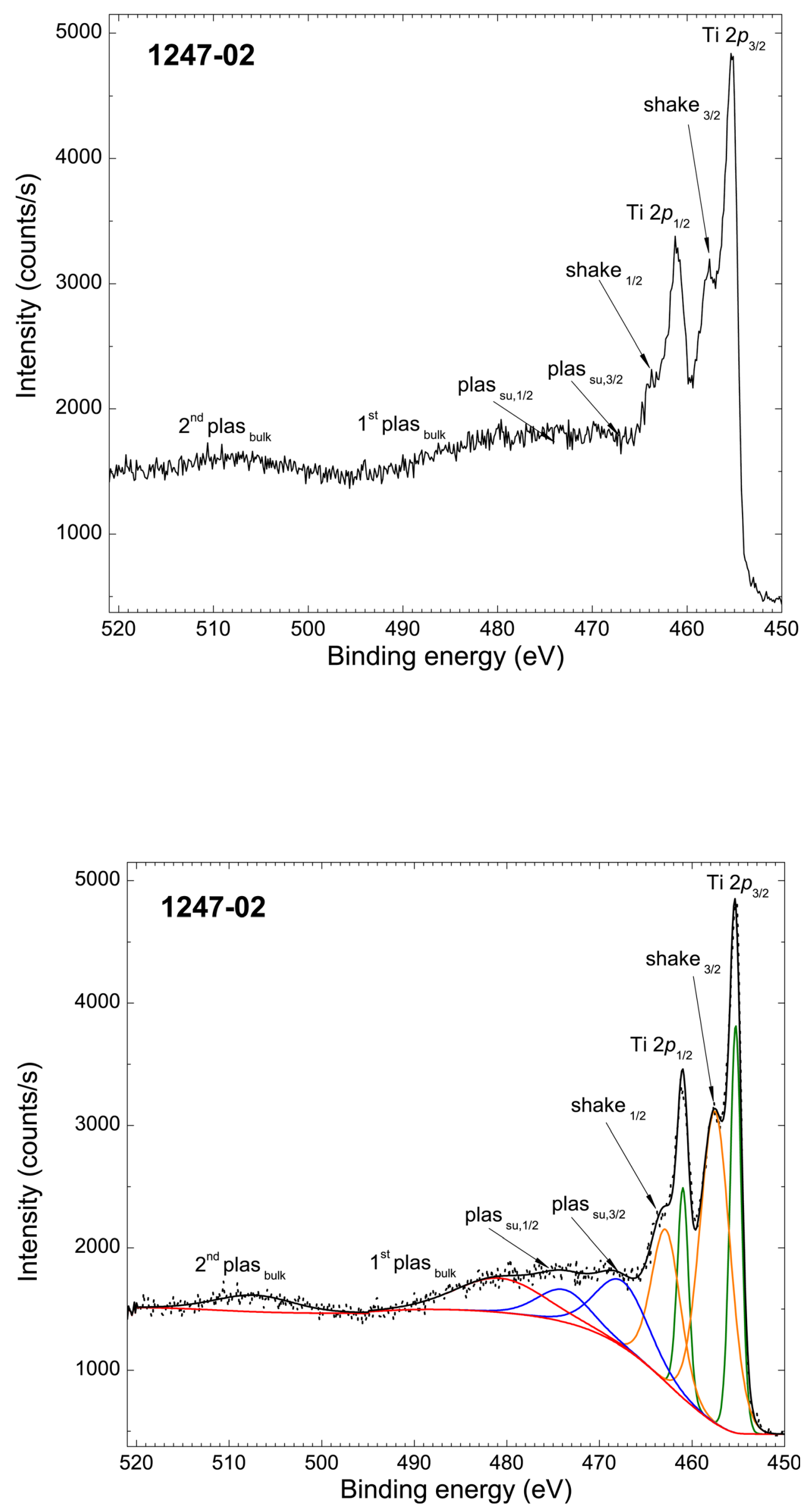


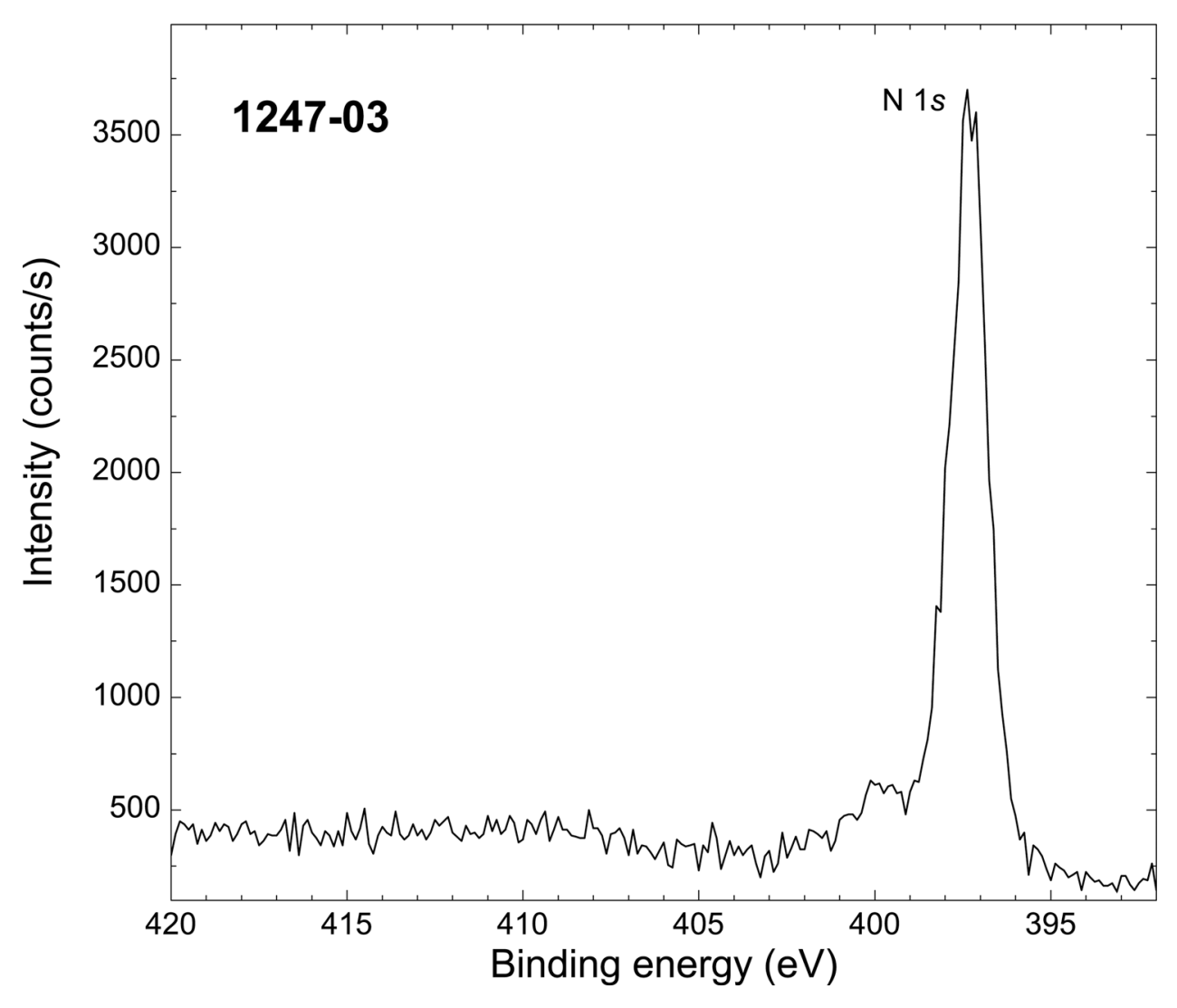

- Accession \#: 01247-03

- Host Material: TiN (001)

- Technique: XPS

- Spectral Region: N $1 s$

Instrument: Phi-Instrument Quantum 2000

Excitation Source: Al $K_{\alpha}$ monochromatic

Source Energy: $1486.6 \mathrm{eV}$

Source Strength: $25 \mathrm{~W}$

Source Size: $0.1 \mathrm{~mm} \times 0.1 \mathrm{~mm}$

Analyzer Type: spherical sector

Incident Angle: 0 or $1^{\circ}$

Emission Angle: 45 or $44^{\circ}$

Analyzer Pass Energy: $58.7 \mathrm{eV}$

Analyzer Resolution: $0.125 \mathrm{eV}$

Total Signal Accumulation Time: $336 \mathrm{~s}$

Total Elapsed Time: $\sim 380 \mathrm{~s}$

Number of Scans: 15

Effective Detector Width: $8 \mathrm{eV}$

Analyzer Angular Acceptance Width: $20^{\circ} \times 20^{\circ}$

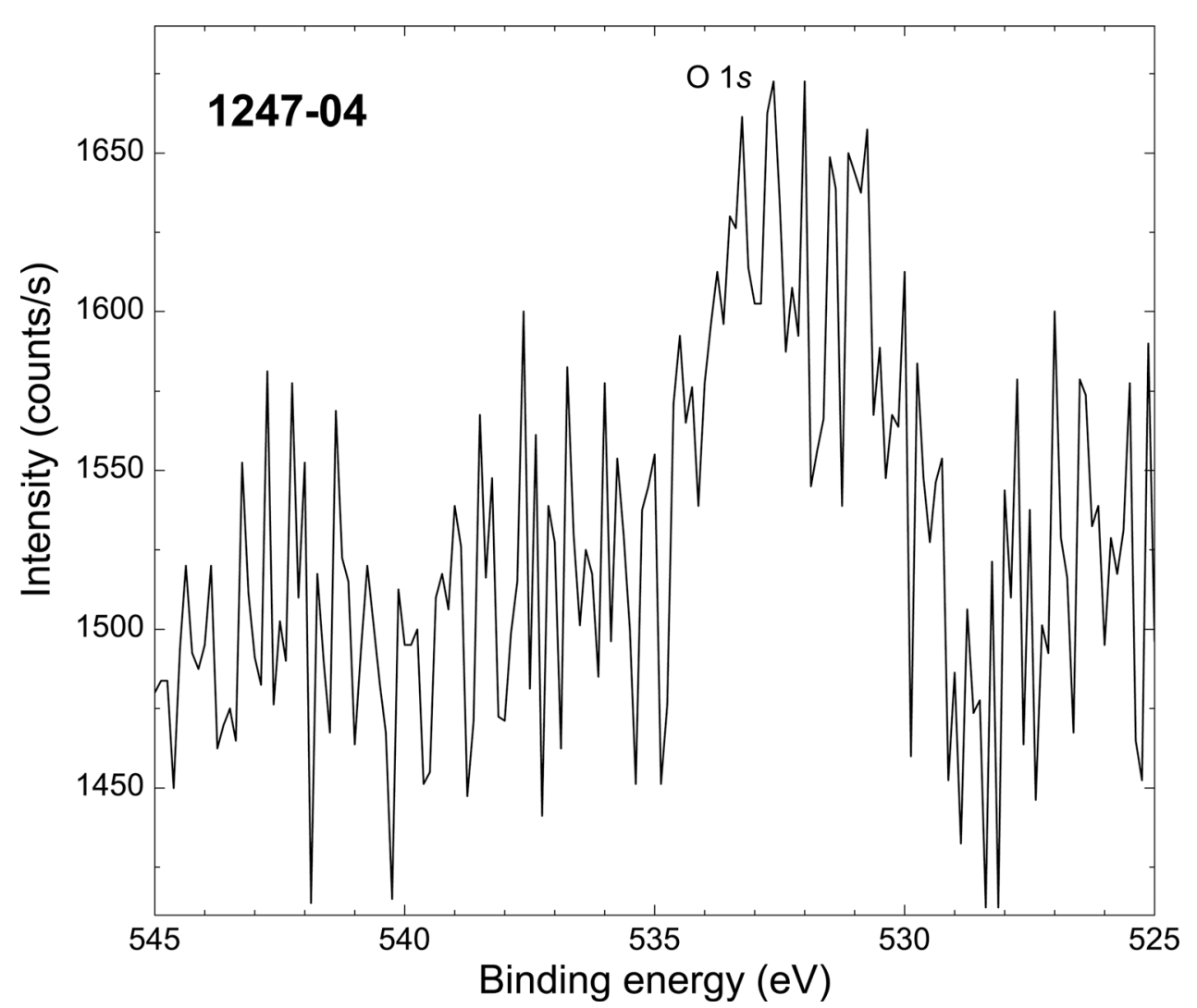

Accession \#: 01247-04

- Host Material: TiN (001)

- Technique: XPS

- Spectral Region: $01 \mathrm{~s}$

Instrument: Phi-Instrument Quantum 2000

Excitation Source: Al $K_{\alpha}$ monochromatic

Source Energy: $1486.6 \mathrm{eV}$

Source Strength: $25 \mathrm{~W}$

Source Size: $0.1 \mathrm{~mm} \times 0.1 \mathrm{~mm}$ Analyzer Type: spherical sector Incident Angle: 0 or $1^{\circ}$

Emission Angle: 45 or $44^{\circ}$

Analyzer Pass Energy: $58.7 \mathrm{eV}$

Analyzer Resolution: $0.125 \mathrm{eV}$

Total Signal Accumulation Time: $51 \mathrm{~s}$ Total Elapsed Time: $90 \mathrm{~s}$

Number of Scans: 4

Effective Detector Width: $8 \mathrm{eV}$

Analyzer Angular Acceptance Width: $4^{\circ} \times 4^{\circ}$ 


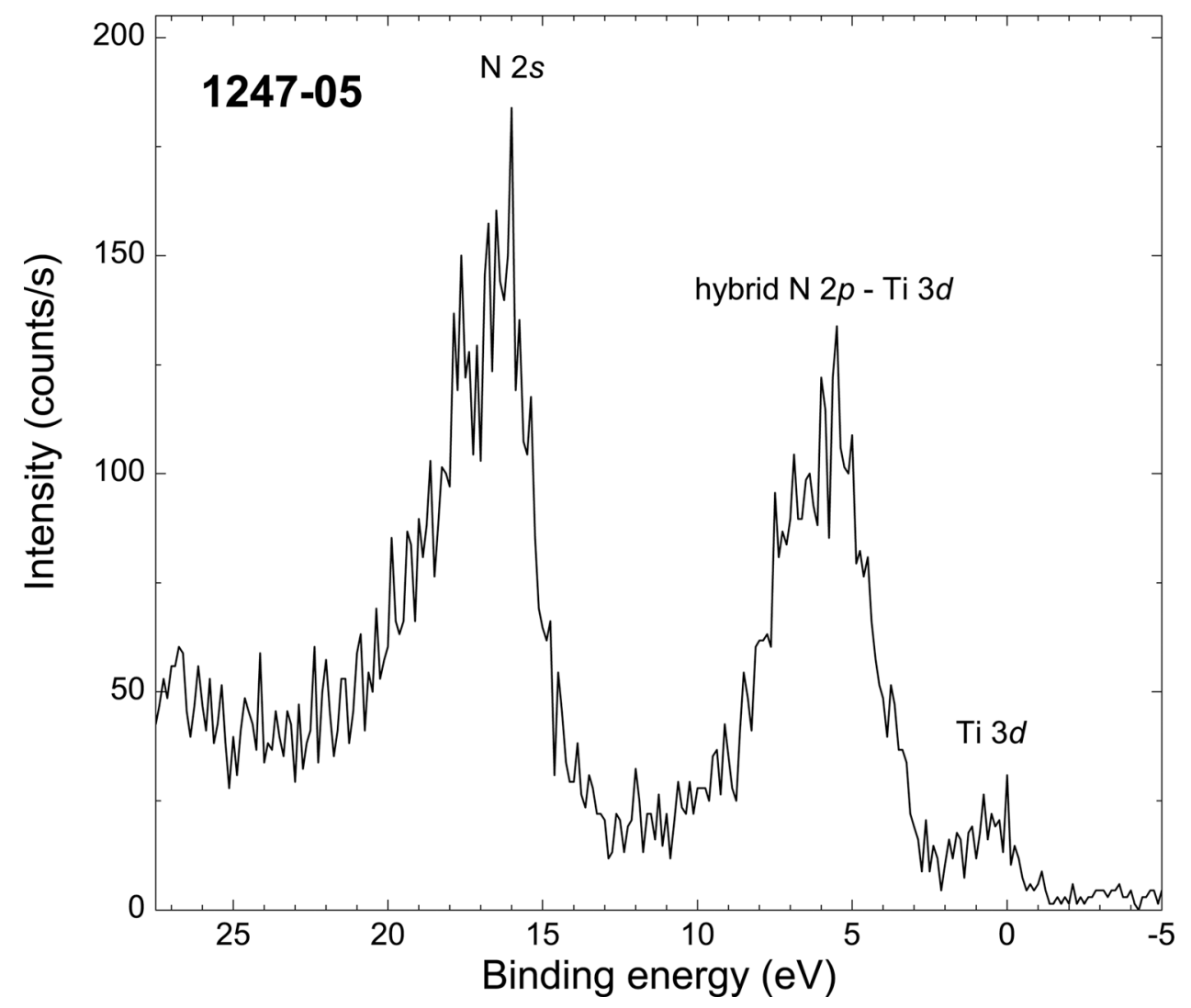

Accession \#: 01247-05

- Host Material: TiN (001)

- Technique:XPS

- Spectral Region: valence band

Instrument: Phi-Instrument Quantum 2000

Excitation Source: Al $K_{\alpha}$ monochromatic

Source Energy: $1486.6 \mathrm{eV}$

Source Strength: $25 \mathrm{~W}$

Source Size: $0.1 \mathrm{~mm} \times 0.1 \mathrm{~mm}$ Analyzer Type: spherical sector Incident Angle: 0 or $1^{\circ}$

Emission Angle: 45 or $44^{\circ}$

Analyzer Pass Energy: $58.7 \mathrm{eV}$

Analyzer Resolution: $0.125 \mathrm{eV}$

Total Signal Accumulation Time: not specified

Total Elapsed Time: not specified

Number of Scans: 20

Effective Detector Width: $8 \mathrm{eV}$

Analyzer Angular Acceptance

Width: $4^{\circ} \times 4^{\circ}$

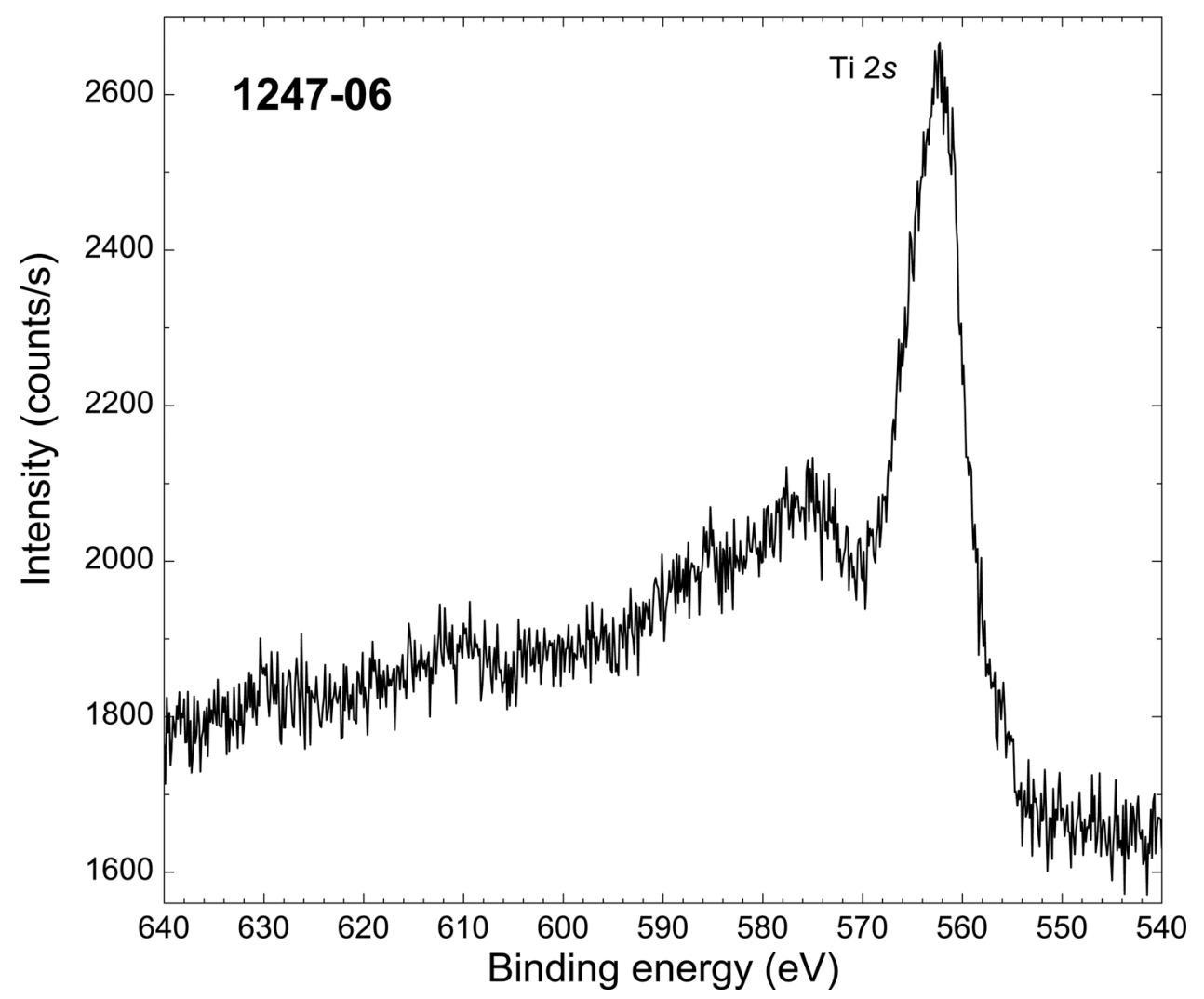

Accession \#: 01247-06

- Host Material: TiN (001)

- Technique: XPS

- Spectral Region: Ti $2 s$

Instrument: Phi-Instrument Quantum 2000

Excitation Source: Al $K_{\alpha}$ monochromatic

Source Energy: $1486.6 \mathrm{eV}$

Source Strength: $25 \mathrm{~W}$

Source Size: $0.1 \mathrm{~mm} \times 0.1 \mathrm{~mm}$

Analyzer Type: spherical sector

Incident Angle: 0 or $1^{\circ}$

Emission Angle: 45 or $44^{\circ}$

Analyzer Pass Energy: $58.7 \mathrm{eV}$

Analyzer Resolution: $0.125 \mathrm{eV}$

Total Signal Accumulation Time: $900 \mathrm{~s}$

Total Elapsed Time: $3600 \mathrm{~s}$

Number of Scans: 17

Effective Detector Width: $8 \mathrm{eV}$

Analyzer Angular Acceptance Width: $20^{\circ} \times 20^{\circ}$ 


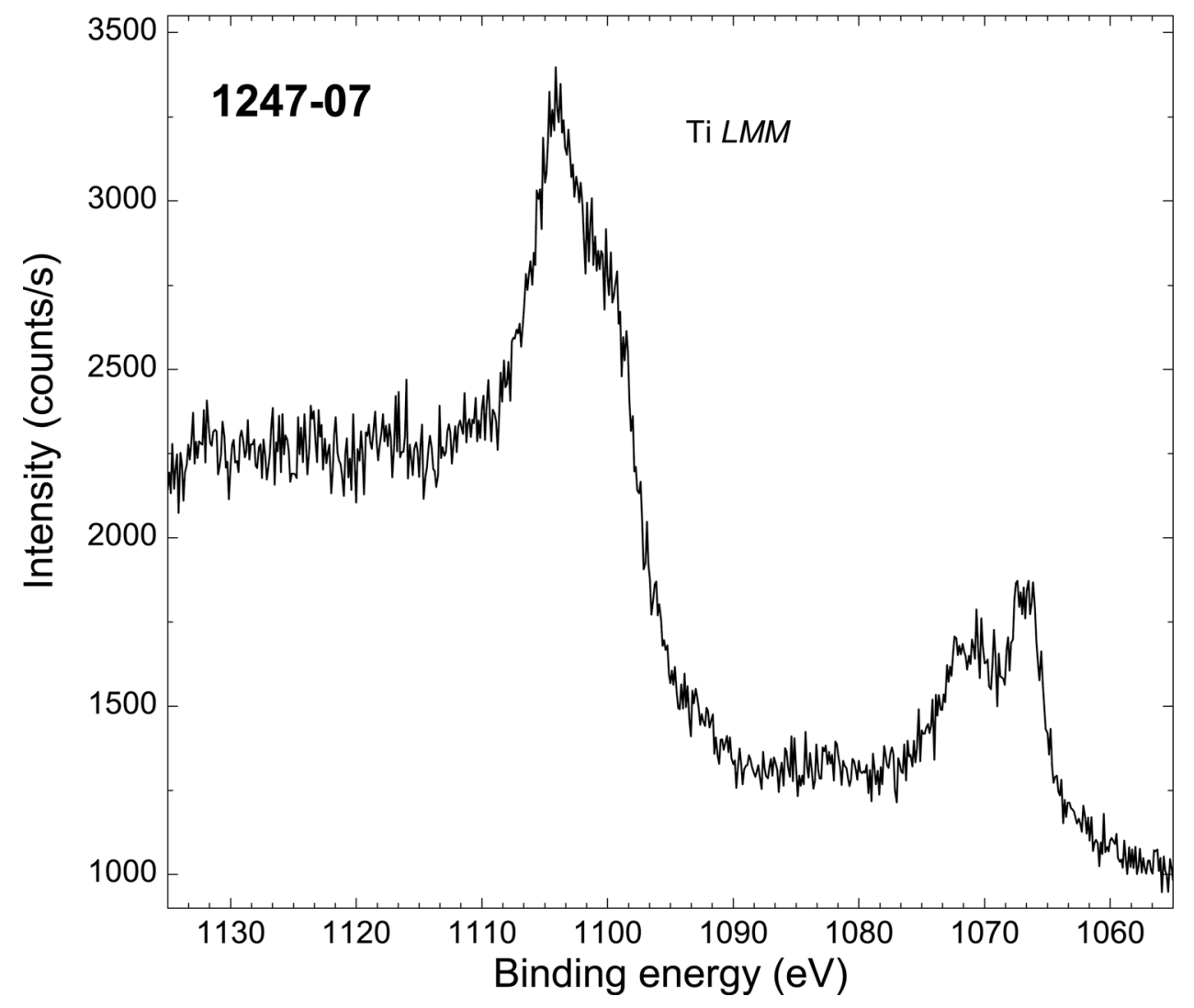

Accession \#: 01247-07

- Host Material: TiN (001)

Technique: XPS

- Spectral Region: Ti $L M M$

Instrument: Phi-Instrument Quantum 2000

Excitation Source: Al $K_{\alpha}$ monochromatic

Source Energy: $1486.6 \mathrm{eV}$

Source Strength: $25 \mathrm{~W}$

Source Size: $0.1 \mathrm{~mm} \times 0.1 \mathrm{~mm}$

Analyzer Type: spherical sector Incident Angle: 0 or $1^{\circ}$

Emission Angle: 45 or $44^{\circ}$

Analyzer Pass Energy: $58.7 \mathrm{eV}$

Analyzer Resolution: $0.125 \mathrm{eV}$

Total Signal Accumulation Time: $900 \mathrm{~s}$

Total Elapsed Time: $3600 \mathrm{~s}$

Number of Scans: 17

Effective Detector Width: $8 \mathrm{eV}$

Analyzer Angular Acceptance Width: $20^{\circ} \times 20^{\circ}$ 